\title{
Numerical Simulation on Flow Field of Oilfield Three-Phase Separator
}

\author{
Yong-tu Liang, ${ }^{1}$ Sheng-qiu Zhao, ${ }^{1,2}$ Xia-xue Jiang, ${ }^{1}$ Xian-qi Jia, ${ }^{1}$ and Wang Li ${ }^{1}$ \\ ${ }^{1}$ Beijing Key Laboratory of Urban Oil and Gas Distribution Technology, China University of Petroleum, Beijing 102249, China \\ ${ }^{2}$ Trans-Asia Gas Pipeline Company Limited, Beijing 100007, China \\ Correspondence should be addressed to Yong-tu Liang; liangyt21st@163.com
}

Received 26 March 2013; Accepted 13 April 2013

Academic Editor: Bo Yu

Copyright (c) 2013 Yong-tu Liang et al. This is an open access article distributed under the Creative Commons Attribution License, which permits unrestricted use, distribution, and reproduction in any medium, provided the original work is properly cited.

\begin{abstract}
The conventional measurement method can no longer guarantee the accuracy requirement after the oilfield development entering high water cut stage, due to the water content and gas phase in the flow. In order to overcome the impact of measurement deviation the oilfield production management, the flow field of three-phase separator is studied numerically in this paper using Fluent 6.3.26. Taking into consideration the production situation of PetroChina Huabei Oilfield and the characteristics of three-phase separator, the effect of internal flow status as well as other factors such as varying flow rate, gas fraction, and water content on the separation efficiency is analyzed. The results show that the separation efficiencies under all operation conditions are larger than $95 \%$, which satisfy the accuracy requirement and also provide the theoretical foundation for the application of three-phase separators at oilfields.
\end{abstract}

\section{Introduction}

The rapid development of national economy has greatly increased the consumption of petroleum of China. Since China became net importer of petroleum in 1993, the import dependence has raised from $6 \%$ to $55.2 \%$ in 2010 [1-3]. The pressing demand for oil has led to the consecutive production of China's interior oilfield during high water cut stage. In the daily operation management of oilfields, the accurate and timely measurement of well fluids is very important, which would become the significant basis for the supervision of operation, the analysis of reservoir change, and the establishment of production decisions. Up to now, the measurement method for wells at PetroChina Huabei Oilfield is sampling. This kind of traditional measurement method can no longer guarantee the precision during high water cut stage due to the extensive variation of water content and gas-oil ratio, bringing much inconvenience to the operation management. In order to overcome this, it is necessary to select a threephase separate measurement system, which could provide the accurate measurement as well as the automatic control of well fluids.
The former numerical studies of interior flow field of separator concentrate on gravity separator and centrifugal separator, and a great many of them focus on specific work conditions. The physical model and calculation method will vary the work conditions.

Yang et al. [4] conducted the numerical simulation of gravity separator by CFD method, analyzed the influence of separation factors on separating efficiency, and believed the most important factors are that diameter of gas particles and liquid viscosity, followed by gas content and throughput. Luo et al. [5] introduced RSM model to obtain the velocity, pressure, and concentration distribution of the [gas-liquid measure cyclone], which provided basis for the improvement and optimization of separator structure. Zhou and Xia [6] analyzed the impact of centrifugal force as well as the collision process of air stream at the wall on separation effectiveness and proposed a remodel design scheme based on this gasliquid two-phase simulation. Du [7] increased the measuring precision of crude oil (and associated water) and associated gas to $0.5 \%$ and $5 \%$, respectively, by adopting new measuring technology which included the combined application of mass flow meter, intelligent gas flow meter, and microcomputer. 


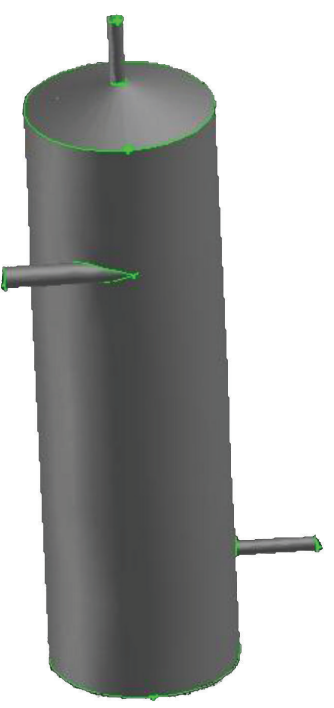

FIgURE 1: Model of three-phase separator.

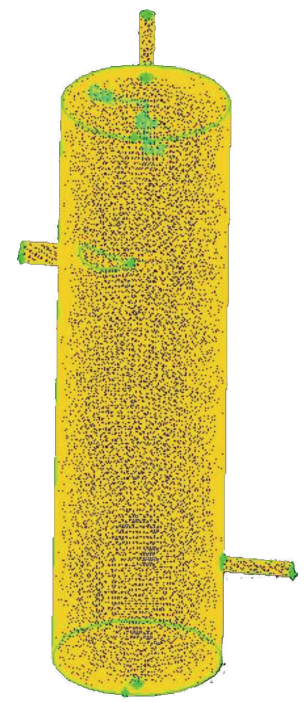

FIgURe 2: Mesh system.

Based on low permeability oilfield development and production situation, Pan et al. [8] designed an XYD oilgas-water three-phase automatic metering unit which could realize the automatic online consecutive metering of oilgas-water [and the gas production, well production of a single well.] Pang et al. [9] combined cyclone separator and horizontal separator together, manufacturcing an XJ oil-gaswater three-phase metering unit especially for high water cut oilfields, which is able to improve the gas-liquid separation effectively. Qu and $\mathrm{Ma}$ [10] employed cyclone separator and stabilization tank simultaneously for separation and designed a skid-mounted three-phase separating and metering unit. Oil-gas-water three-phase metering is achieved by the joint use of mass flow meter and gas vortex precession flow meter. This unit is portable, effective, and compact and is suitable for single well metering and calibration.

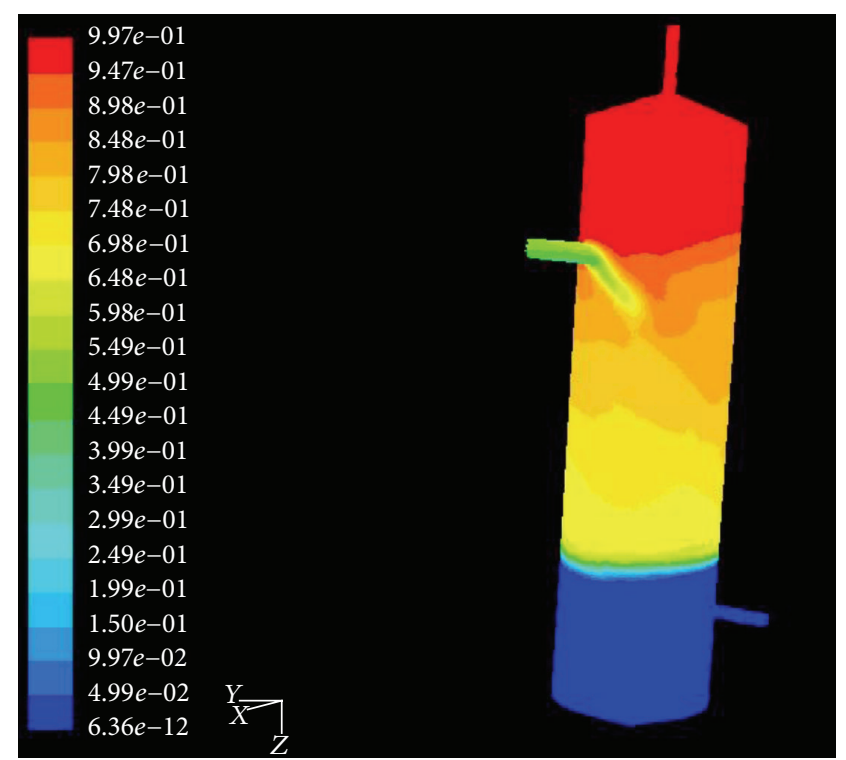

FIGURE 3: Distribution of volume fraction of gas.

In this paper, the process of oil-gas-water three-phase separation by gravity vertical metering separator is numerically simulated by CFD methods. Internal flow status and the effect of factors such as varying flow rate, gas ratio, and water ratio on the separation efficiency are analyzed, which may provide the theoretical foundation for the application of three-phase separators at oilfields.

\section{Methodology}

2.1. Physical Model. The three-phase separator calculated in this study is $4.4 \mathrm{~m}$ high, the diameter of which is $1.2 \mathrm{~m}$. The inlet diameter is $0.159 \mathrm{~m}$ and the inlet is $0.8 \mathrm{~m}$ below the top. The diameters of gas outlet and liquid outlet are both $0.1 \mathrm{~m}$. Liquid outlet is $0.6 \mathrm{~m}$ above the bottom. As shown in Figure 1, the geometric model of this separator is achieved by Gambit. The inlet of separator is at the tangential, which means that it is spiral-flow-type inlet diverter. Mesh is generated by Tex/Hybrid scheme, the interval size of which is 0.5. Total amount of grid number is 260,000 . The mesh system is shown in Figure 2. The multiphase flow model employed in this paper is VOF, while RNG $k-\varepsilon$ model is adopted for the calculation of turbulent flow.

\subsection{Boundary Conditions and Parameters}

2.2.1. Inlet Boundary. Normally, there are three kinds of inlet boundary conditions, which are mass inlet, pressure inlet, and velocity inlet. Mass inlet and velocity inlet show no difference for incompressible fluids. When inlet velocity or flow rate is unknown, pressure inlet should be employed by giving gross pressure and static pressure.

The velocity boundary is adopted. The flow rate, water content, and gas content are considered as variables since the incoming fluid is unstable at the metering station. 


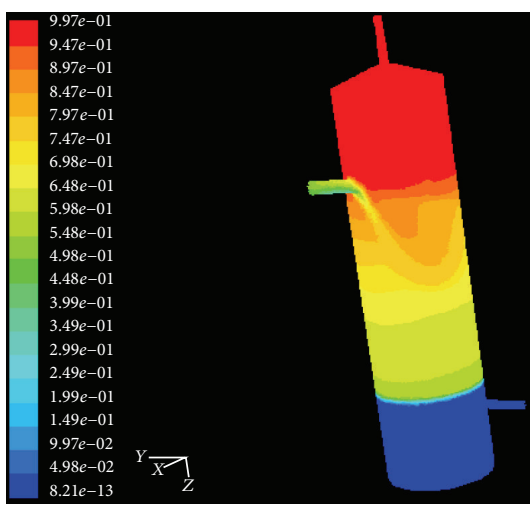

(a) Case 1

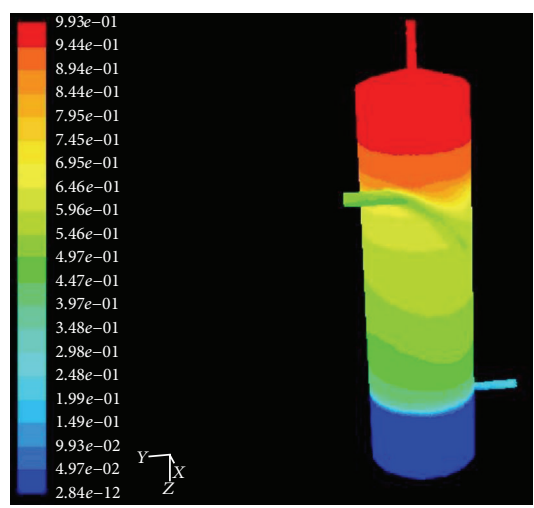

(c) Case 4

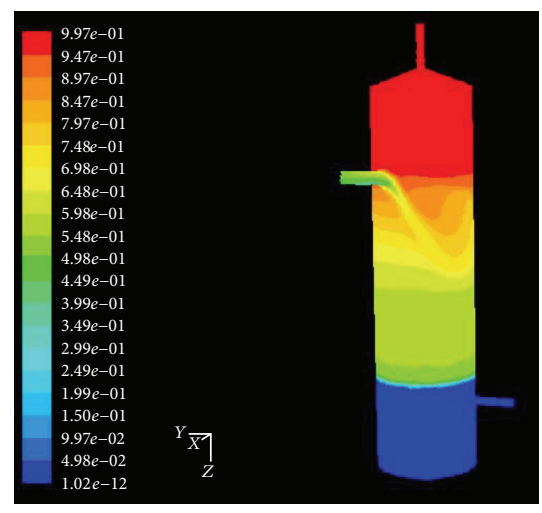

(b) Case 2

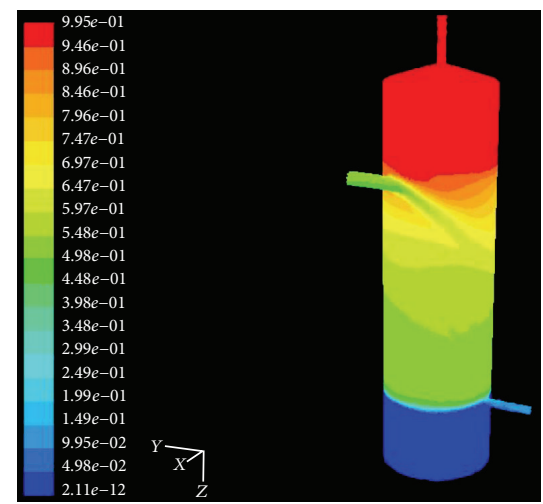

(d) Case 5

FIGURE 4: Results of flow distribution under different flow rates.

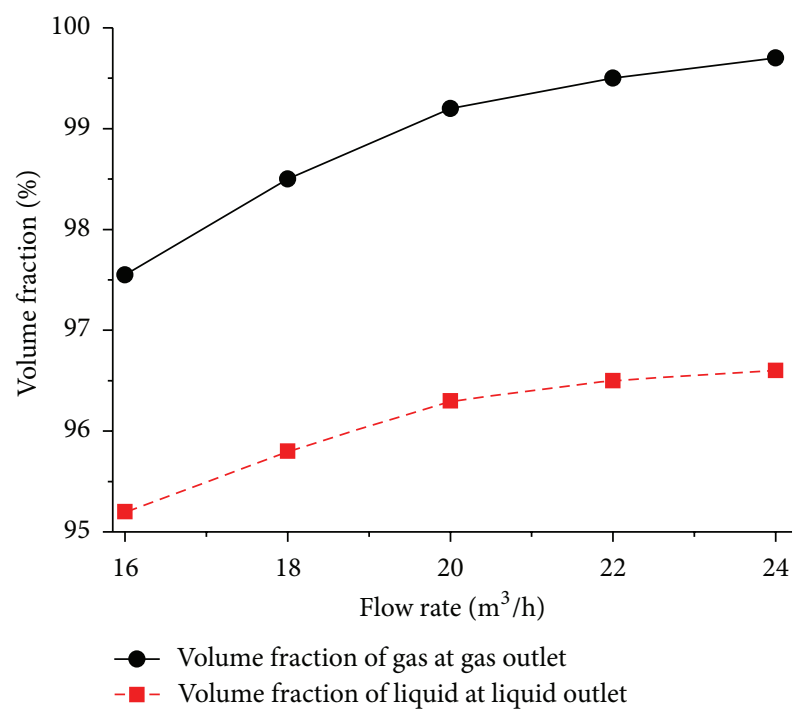

Figure 5: Impact of flow rate on the separation efficiency.

2.2.2. Outlet Boundary. There are many kinds of outlet boundary conditions, among which flow rate and pressure condition are the most commonly used. The outlet boundary condition cannot be chosen well, but should be set according to the inlet. In this case, the outlet velocity is calculated by continuity equation by considering inlet flow rate.

2.2.3. Wall Interface. In the condition of this study, the wall of the separator is set, and the medium inside is viscous, making it a nonslip boundary condition. Besides this, other parameters such as roughness, thermal property, and permeability property are all defined as default in Fluent.

2.2.4. Parameters. The densities of crude oil, water, and natural gas are $890 \mathrm{~kg} / \mathrm{m}^{3}, 1000 \mathrm{~kg} / \mathrm{m}^{3}$, and $0.6679 \mathrm{~kg} / \mathrm{m}^{3}$, respectively, and the kinetic viscosities of them are $0.093 \mathrm{~kg} /(\mathrm{m} \cdot \mathrm{s})$, $0.001 \mathrm{~kg} /(\mathrm{m} \cdot \mathrm{s})$, and $12.28 \times 10^{-5} \mathrm{~kg} /(\mathrm{m} \cdot \mathrm{s})$, respectively. The diameter of droplet is $100 \mu \mathrm{m}$. The operation pressure and temperature are $0.3 \mathrm{MPa}$ and $48^{\circ} \mathrm{C}$.

\section{Results and Analyses}

After numerical calculation of oil-gas-water three-phase separator, the internal flow status and the impact of varying flow rate, gas ratio, and water ratio are analyzed, so as to figure out the feasibility of this kind of separator for PetroChina Huabei Oilfield.

3.1. Flow Field. It is the turbulent multiphase flow at the inlet of the separator. In this case, the flow rate is $20 \mathrm{~m}^{3} / \mathrm{h}$ with 


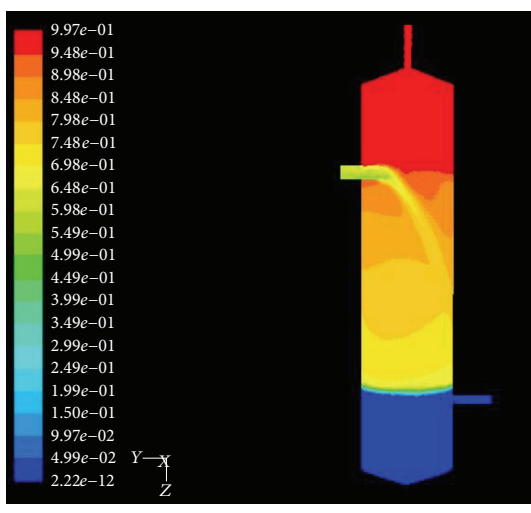

(a) Case 1

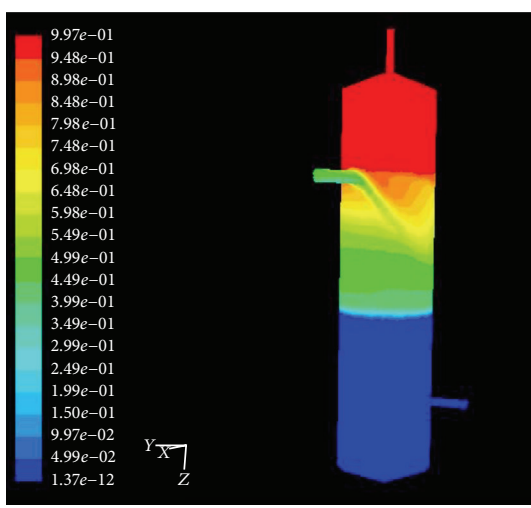

(c) Case 4

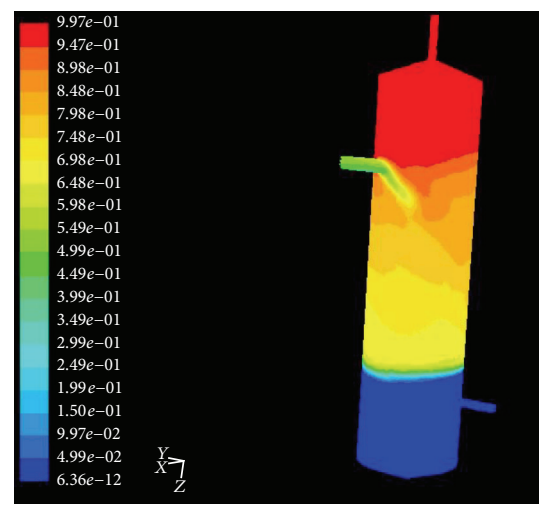

(b) Case 2

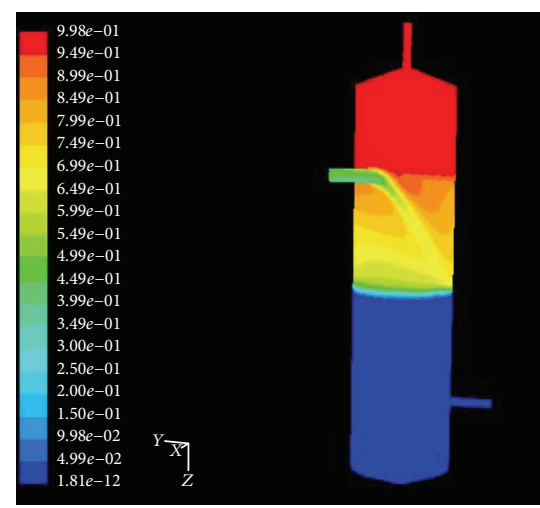

(d) Case 5

FIGURE 6: Results of flow distribution under different gas volume fractions.

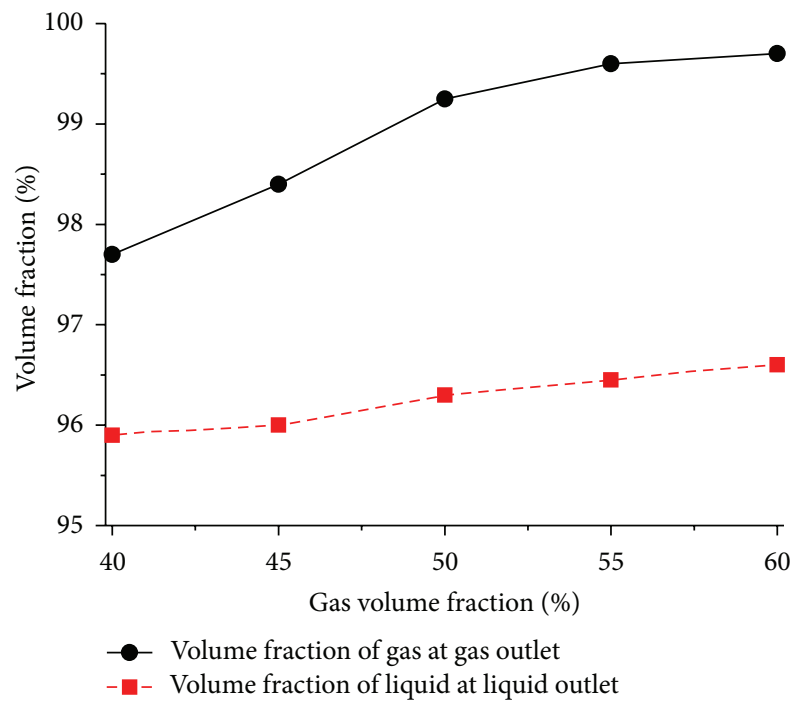

FIGURE 7: Impact of gas volume fraction on the separation efficiency.

the water and gas contents of $80 \%$ and $50 \%$. The result is shown as follows.

As Figure 3 shows, fluids flow down rotationally due to the kinetic energy and gravity. The separation is proceeded in the separation region, in which the gas fraction is
TABLE 1: Flow rate of different cases.

\begin{tabular}{lccccc}
\hline Case & 1 & 2 & 3 & 4 & 5 \\
Flow rate $\left(\mathrm{m}^{3} / \mathrm{h}\right)$ & 16 & 18 & 20 & 22 & 24 \\
\hline
\end{tabular}

$50 \%-80 \%$, apparently displaying gradient along the gravity direction. Gas fraction at the liquid-phase region is below $5 \%$. Oppositely, it is above $95 \%$ at the gas-phase region, indicating the effectiveness of separation. This result matches the actual operational condition well, illustrating the viability of selected algorithm, multiphase flow model, and turbulent model.

The separation efficiency is represented by the gas fraction at the gas outlet and the liquid fraction at the liquid outlet. The higher the gas fraction at the gas outlet, the less liquid brought by gas, the better the efficiency and, vice versa.

3.2. Impact of Flow Rate. Flow rate changes frequently in the actual working condition of oil and gas gathering system, which may significantly affect the internal flow distribution of the separator. Meanwhile, the spiral-flow-type inlet diverter makes separation efficiency quite sensitive to the inlet flow rate. In this numerical study, the gas volume fraction and water content are fixed to $50 \%$ and $80 \%$, respectively, while flow rate is changed as Table 1 shows to demonstrate the impact of flow rate. 


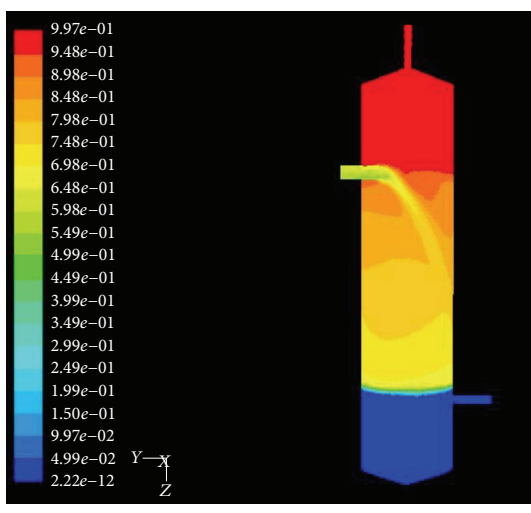

(a) Case 1

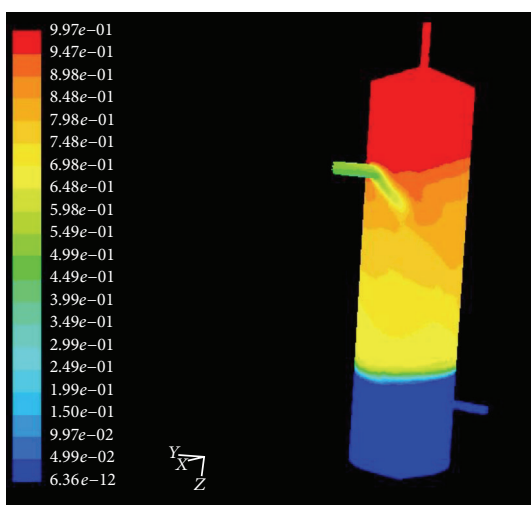

(c) Case 4

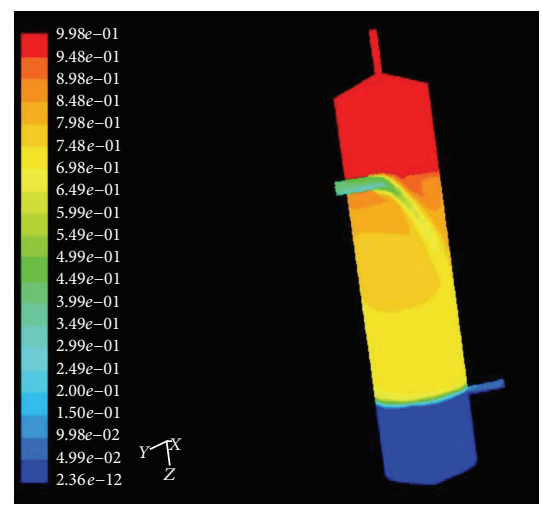

(b) Case 2

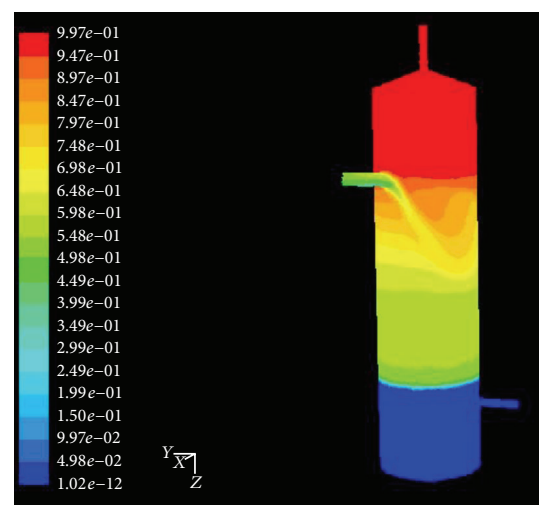

(d) Case 5

FIGURE 8: Results of flow distribution under different water contents.

TABLE 2: Gas volume fraction of different cases.

\begin{tabular}{lccccc}
\hline Case & 1 & 2 & 3 & 4 & 5 \\
Gas volume fraction (\%) & 40 & 45 & 50 & 55 & 60 \\
\hline
\end{tabular}

From the results shown in Figures 3 and 4, the stream line tends to be gentle after fluids entered the separator. And it can be seen that the larger variation of gas volume fraction along the gradient direction in the gas-liquid separation region, the faster the separation. The trends of gas fraction and liquid fraction at the outlet are shown in Figure 5.

Figure 5 shows that, in the actual working load of oilfield production, gas volume fraction at gas outlet and liquid volume fraction at liquid outlet can remain above $97 \%$ and 95\%. And the separation efficiency would be even larger with the increased flow rate. That is because the inlet of the separator is tangent with the main body, and the mixture will be separated by self-rotary motion. The higher the flow rate, the larger the centrifugal force, the better separation effect.

3.3. Impact of Gas Volume Fraction. Another factor that may remarkably affect the separation is gas volume fraction. Considering the lease operation condition, the flow rate and water content are fixed to $20 \mathrm{~m}^{3} / \mathrm{h}$ and $80 \%$, respectively, while the gas volume fraction is changed as Table 2 shows.
TABLE 3: Water content of different cases.

\begin{tabular}{lccccc}
\hline Case & 1 & 2 & 3 & 4 & 5 \\
Water content (\%) & 64 & 72 & 80 & 88 & 96 \\
\hline
\end{tabular}

As can be seen from Figure 6, with the increase of gas volume fraction, the gas-liquid separation region is getting smaller. Moreover, the separation process will be accelerated if there is large gas volume fraction gradient along the vertical direction.

Figure 7 indicates that gas volume fraction at gas outlet and liquid volume fraction at liquid outlet can remain above $97 \%$ and $96 \%$, and they will increase with the gas volume fraction. This can demonstrate that spiral-flow separator is more suitable for the separation of mixture with large gas-oil ratio.

3.4. Impact of Water Content. PetroChina Huabei Oilfield is in the high water cut stage, while water flooding is adopted, making the water fraction high among well fluids. The variation of water will have an impact on the viscosity and density of well fluids mixture, thus affecting the flow condition in the metering separator, further influencing the separation efficiency. In this part, the flow rate is set to $20 \mathrm{~m}^{3} / \mathrm{h}$ and gas volume fraction is $50 \%$, with the water content varying as Table 3 shows. 


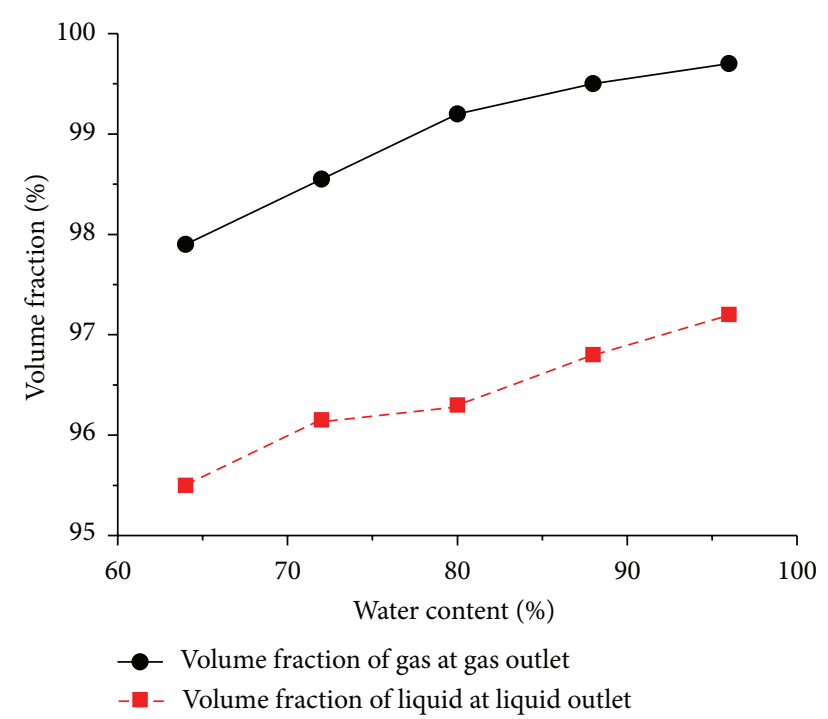

FIGURE 9: Impact of water content on the separation efficiency.

It can be easily seen from Figure 8, with the increasing water content, in the gas-liquid separation region, that gas volume fraction gradient along the vertical direction becomes larger, indicating better separation effect.

The impact of water content on the separation efficiency is shown in Figure 9. With the gas volume fraction at gas outlet and the liquid volume fraction at liquid outlet remaining above $97 \%$ and $95 \%$, the separation efficiency would increase with higher water content. The reason for this phenomenon is that increasing water content leads to the decrease of mixture viscosity, which is beneficial to the flow of mixture in the separator. Besides, larger water content gives smaller ratio of crude oil, making it easier for natural gas to separate.

\section{Conclusions}

Based on the previous results and analyses, it is reasonable to conclude that

(1) confined to the production flow rate range of oilfield, the efficiency of metering separator would increase with the augmentation of well fluids flow rate;

(2) spiral-flow diverter is propitious to large gas-oil ratio mixture, and large gas volume fraction would contribute to the separation efficiency;

(3) the increasing water content would significantly reduce the viscosity of mixture, so as to promote the separation, and meanwhile, the natural gas could be easier to separate since the oil ratio decreased.

After the analyses of factors such as varying flow rate, gas fraction, and water content to the separation efficiency, the gas volume fraction at gas outlet and the liquid volume fraction at liquid outlet can remain above $97 \%$ and $95 \%$, respectively, providing clear evidence of the feasibility of this kind of three-phase separate measurement system at PetroChina Huabei Oilfield.

\section{Acknowledgment}

The study is supported by the National Science Foundation of China (no. 51276198).

\section{References}

[1] L. Zhang, Z. Yang, J. Liang, and Y. Cai, "Spatial variation and distribution of urban energy consumptions from cities in China," Energies, vol. 4, no. 1, pp. 26-38, 2011.

[2] Q. Zhai, H. Cao, X. Zhao, and C. Yuan, "Cost benefit analysis of using clean energy supplies to reduce greenhouse gas emissions of global automotive manufacturing," Energies, vol. 4, no. 10, pp. 1478-1494, 2011

[3] X. Dong, Y. Zhang, W. Cui et al., "Emergy-based adjustment of the agricultural structure in a low-carbon economy in Manas County of China," Energies, vol. 4, no. 9, pp. 1428-1442, 2011.

[4] Q. Yang, J. Feng, H. B. Luo et al., "CFD simulation of the inner flow field of the gas-liquid gravity separator," Mechanical Research \& Application, vol. 20, no. 2, pp. 72-74, 2007.

[5] H. B. Luo, J. Feng, Q. Yang et al., "CFD simulation of the inner flow field of the gas-liquid measure cyclones," Mechanical Engineer, vol. 1, pp. 74-76, 2007.

[6] H. Zhou and N. Xia, "Numerical simulation on the gasliquid flows inside the oil-gas Separators," Chinese Journal of Computational Mechanics, vol. 23, no. 6, pp. 766-771, 2006.

[7] H. Du, "Research and application of the measurement technology about the three-phase fluid about oil well," Fault-Block Oil ¿ Gas Field, vol. 12, no. 4, pp. 54-55, 2005.

[8] M. Y. Pan, Y. J. Geng, B. H. Huang et al., "Application of XDY oil-gas-water three-phase metering unit," Oil-Gasfield Surface Engineering, vol. 26, no. 11, pp. 38-39, 2007.

[9] H. Pang, Y. J. Wang, B. H. Huang et al., "YJ oil-gas-water threephase metering unit," Oil-Gasfield Surface Engineering, vol. 28, no. 5, pp. 91-92, 2009.

[10] X. J. Qu and C. J. Ma, "Design of a skid-mounted three-phase separating and metering unit," Automation in Petro-Chemical Industry, vol. 2, pp. 58-60, 2009. 


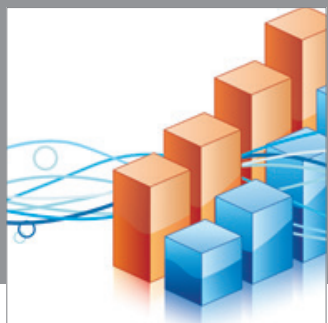

Advances in

Operations Research

mansans

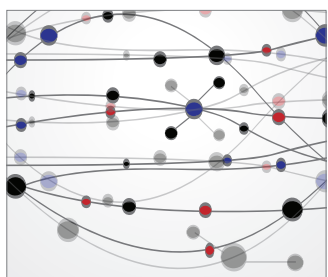

The Scientific World Journal
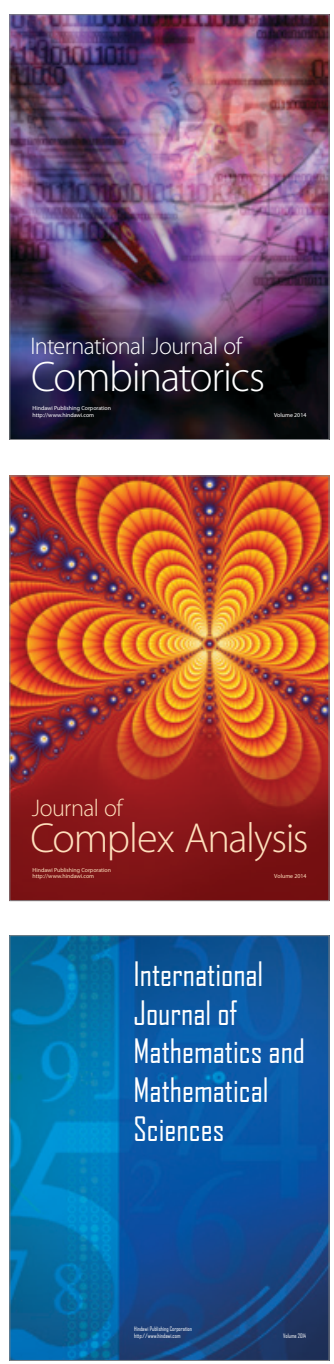
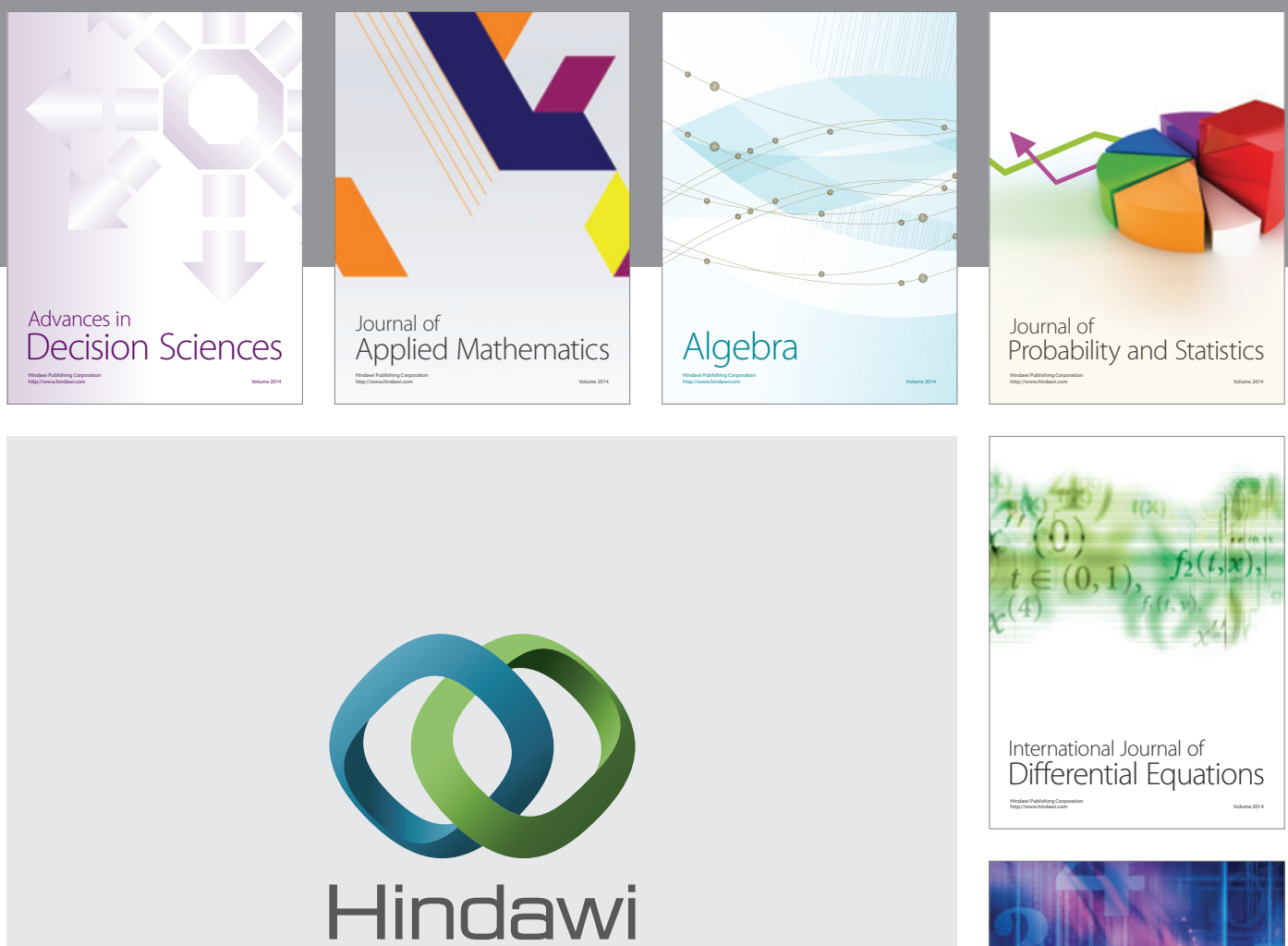

Submit your manuscripts at http://www.hindawi.com
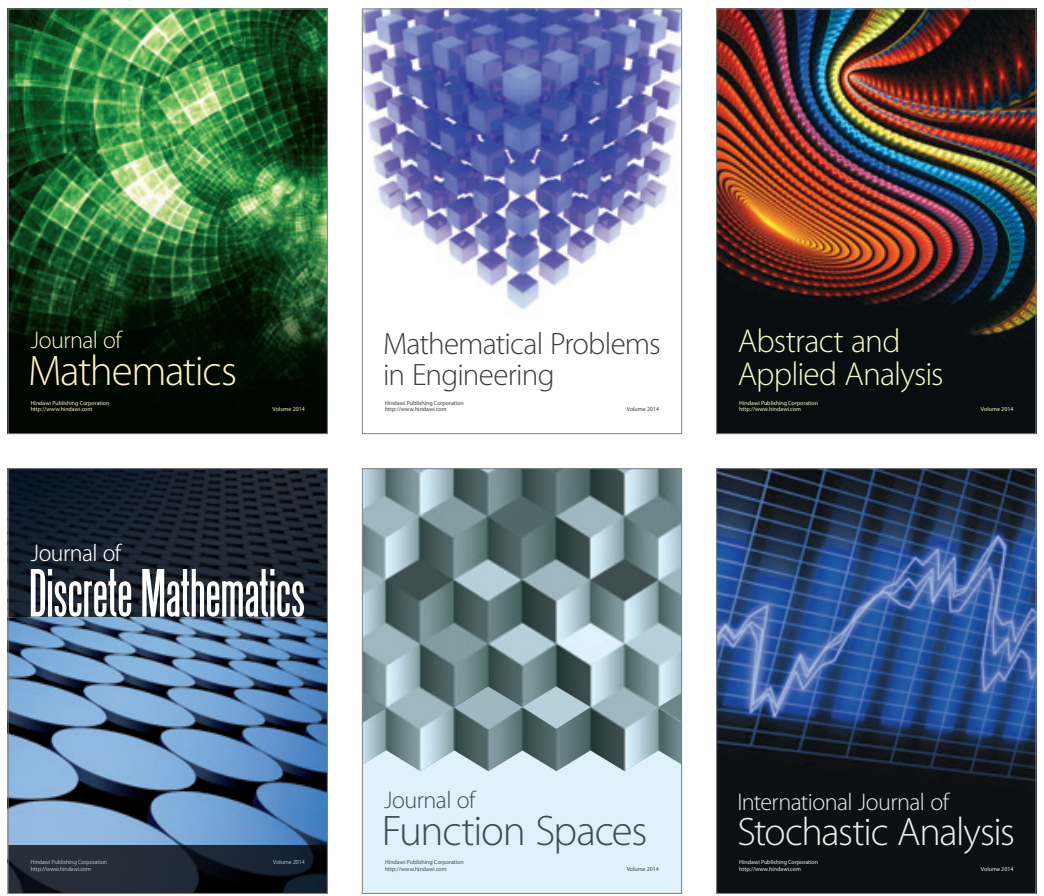

Journal of

Function Spaces

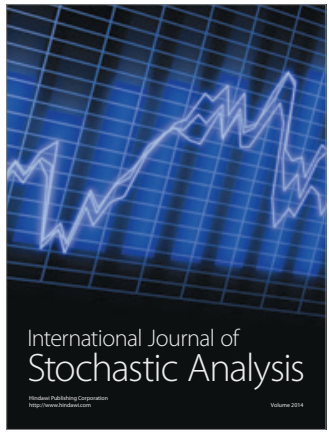

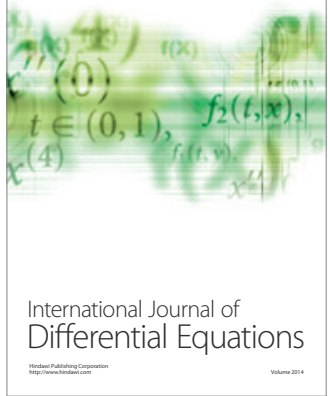
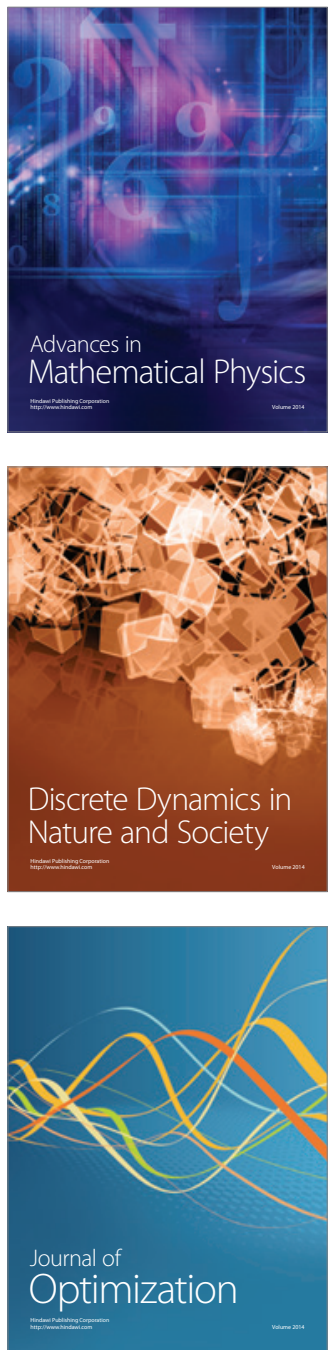